\title{
Diffusion Tensor Imaging of the Brain: Background and Review of Clinical Applications
}

\author{
P. C. Sundgren \\ Department of Radiology, University of Michigan Health System, Ann Arbor, MI, USA
}

Correspondence to:

Pia C. Maly Sundgren, MD, PhD

Associate Professor, Department of Radiology, University of Michigan Health Systems, 1500 E. Medical Center Drive, Ann Arbor, MI 481090030, USA

Tel: +1 734615 2890; Fax: +1 734764 2412; E-mail: sundgren@umich.edu

Key words: Magnetic resonance imaging, diffusion tensor imaging, background, clinical applications, brain.

\section{Summary}

This article reviews the basic theoretical background to diffusion tensor imaging (DTI) and some of the common clinical applications of DTI as of today such as cerebral ischaemia, brain maturation, ageing and cognitive impairment. The value of DTI in the work-up of brain tumours, as a tool in the differentiation of radiation injury versus recurrent tumour, and in brain injury is reviewed. The potential use of this technique in diseases such as epilepsy, multiple sclerosis, Alzheimer's disease and metabolic disorders is also briefly reviewed.

\section{Introduction and background to diffusion tensor imaging}

Diffusion-weighted imaging (DWI) is a well-established method that is implemented as a part of routine protocol at many institutions around the world. DWI measures the diffusivity of visible water molecules (1) and the principles for the measurement of diffusion with magnetic resonance imaging (MRI) are well described $(2,3)$. DWI has shown to be a very useful tool for detection of early signs of ischaemia $(4,5)$, but is also increasingly used in the evaluation of other brain diseases, e.g. multiple sclerosis (6-8), trauma (9, 10), brain tumour $(11,12)$, and hypertensive encephalopathy $(13,14)$, among others.

Further improvements in the imaging of water diffusion have been done by the recent development of the more complex diffusion tensor imaging (DTI) which allows direct examination, in vivo, of some aspects of tissue microstructure. DTI yields quantitative measures reflecting the integrity of white matter fibres tracts by taking advantage of the intrinsic properties of directionality of water diffusion in human brain tissue. The diffusion of water molecules is characterized by Brownian motion. When water molecules are unconstrained, the direction of motion of a given molecule is random. The displacements of water molecules over time are described by a Gaussian distribution. The diffusion is called isotropic when the motion is equal and unconstrained in all directions. However, the microstructure of brain tissue forms physical boundaries that limit the Brownian motion of water molecules, resulting in a restriction of the total amount of diffusion. In microstructures like the white matter fibres, the diffusion of water molecules will be relatively more restricted perpendicular than parallel to the microstructural boundaries and the diffusion is called anisotropic (15).

In plain DWI MRI, diffusion is described using a scalar parameter - the diffusion coefficient $D$. In tissues, such as grey matter, where the measured apparent diffusivity is merely isotropic, it is sufficient to describe the diffusion characteristics with a single scalar apparent diffusion coefficient (ADC). In the presence of anisotropy, the diffusion can no longer be characterized by a single scalar coefficient but requires a tensor $\underline{\mathrm{D}}$, which fully describes the mobility of the molecules along each direction and the correlation between these directions. The mathematical construct used to characterize anisotropic Gaussian diffusion is a second-order diffusion tensor (16). As the tensor is symmetric, at least six unique elements are required to fully characterize the tensor (3). The tensor can be diagonalized such that only three non-zero elements $\left(\lambda_{1}\right.$, $\lambda_{2}$, and $\lambda_{3}$ ) remain along the diagonal. These elements are known as the eigenvalues. Each eigenvalue is associated with an eigenvector $\left(\varepsilon_{1}, \varepsilon_{2}\right.$ and $\left.\varepsilon_{3}\right)$, where the largest of the three eigenvalues $\left(\lambda_{1}\right)$ corresponds to the primary eigenvector $\varepsilon_{1}$ of the diffusion tensor and describes the principal direction of the diffusion at that point, whereas the two minor eigenvalues, $\lambda_{2}$ and $\lambda_{3}$, give the strength of diffusion along directions orthogonal to the preferred direction.

The main artefacts in obtaining DTI data are the usual artefacts and problems associated with acquiring the DWI data from which the diffusion tensor is estimated or measured. Artefacts such as misregistration of the data due to eddy currents, ghosting due to motion artefacts, and signal loss due to susceptibility variations. Hardware issues such as background gradients, gradient non-linearity and miscalibration also have to be taken in consideration. 
However, improvements in image resolution and reduction of distortion have been made using motion-corrected multishot EPI PROPELLER and SENSE-EPI techniques (17, 18).

Diffusion tensor measurements result in a rich data set. Metrics of diffusion anisotropy can be measured in different ways by applying simple or more complicated mathematical formulas and recalculations using the underlying eigenvectors $(3,4,16,19)$.

A common way to summarize diffusion measurements in the DTI is the calculation of parameter for the overall diffusivity and another parameter that reflects anisotropy. The ADC serves for overall diffusivity and is derived from the trace of the diffusion tensor. Anisotropy is represented by the fractional anisotropy (FA) or alternatively the relative anisotropy (RA). The FA is a measure of the portion of the magnitude of the diffusion tensor due to anisotropy. The RA is derived from a ratio between the anisotropic portion of the diffusion tensor and the isotopic portion. Another commonly used value is the volume ratio (VR) which expresses the relationship between the diffusion ellipsoid volume and the volume of a sphere with radius $\{\lambda\}$ (20). From previous work it is known that both the water ADC and the diffusion anisotropy, differ markedly between paediatric brain and adult brain, and, also, both parameters vary with increasing age and location in the brain (21-23).

The differences between these measurements lie in their sensitivity to anisotropy: FA is more sensitive for low anisotropy values, whereas VR is sensitive to high values of diffusion anisotropy, and RA scales linearly for different levels of anisotropy (3). Both FA and RA are 0.0 for a purely isotropic medium. For higher symmetric anisotropic medium FA tends towards 1, while RA tends towards $\sqrt{ } 2$. Both FA and RA maps can be presented as grey scale images for the purpose of evaluation. As for VR, the range is from 1 (isotropic diffusion) to 0 , therefore, some authors prefer to use (1-VR). In contrast to aforementioned measurements which all represent intravoxel anisotropy, the lattice anisotropy index - another way to evaluate diffusion - measures the intervoxel anisotropy. The lattice measures of diffusion anisotropy allow neighbouring voxels to be considered together in a region of interest, without losing anisotropy effects that result from different fibre orientations across voxels (20).

DTI allows us to look at the anisotropic diffusion within the white matter tracts but is limited in demonstrating the spatial, directional diffusion anisotropy. Therefore, new and or more sophisticated methods to demonstrate diffusion directions such as colour coding and fibre tracking have been proposed (24-27).

By choosing the eigenvector associated with the largest eigenvalue, the principal diffusion direction of the brain structure to be examined can be encoded with colour, resulting in colour-coded maps or directionally encoded FA maps (DEG FA maps). In these colour-encoded maps the fibres have been given different colours (red, green and blue) depending on their different diffusion directions (24) (Fig. 1). The magnitude of the anisotropy, such as FA, can be used as an illumination factor of the calculation of a directionally encoded colour image (24).

In fibre tractography, or fibre tracking, white matter tract directions are mapped under the assumption that in each voxel a measure of the local fibre orientation is measured through DTI. The task of tractography is to sensibly assign mathematical associations between adjacent voxels based on eigenvalue and eigenvector information. In DT-MRI tractography it is assumed that the eigenvector associated with the largest eigenvalue is aligned with the direction of the fibre bundle (25-28).

There are several problems and pitfalls associated with the attempts to evaluate the fibre tract tractography quantitatively. First, all of the usual artefacts and problems associated with DWI can adversely affect fibre tracking artefacts such as misregistration of DWI caused by eddy currents, ghosting due to motion artefacts, and signal loss due to susceptibility variations can all affect the computed tractography (27). Interaction between vectors in the places where the fibres are crossing or 'kissing' or where they (a)

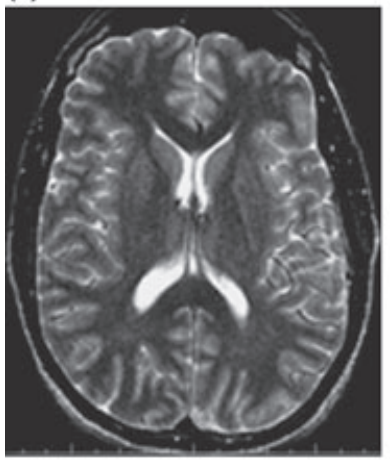

(c)

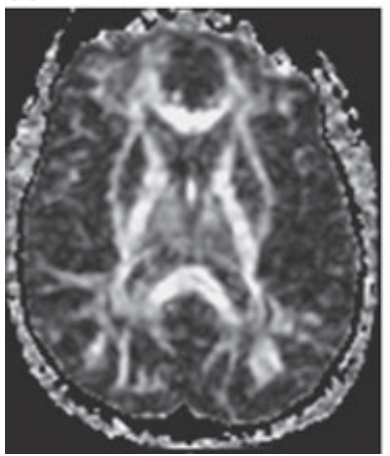

(b)

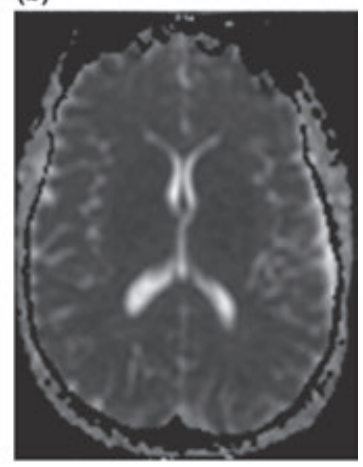

(d)

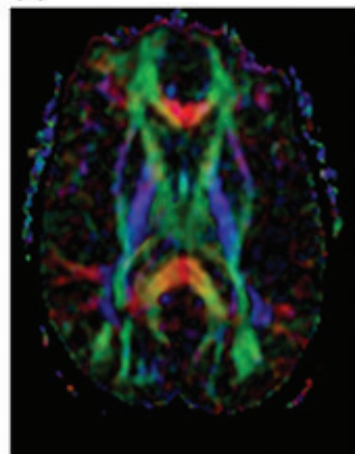

Fig. 1. Normal. A 32-year-old healthy male. Normal T2weighted image (a), apparent diffusion coefficient (ADC) map (b), fractional anisotropy (FA) map (c), and colourcoded FA map (d). On the FA map the highly directional white matter structures are clearly seen (c). On the colourcoded FA map the fibres have been given different colours: blue for superior-inferior, green for anterio-posterior, and red for left to right, depending on their direction of diffusion (d). 
branch or merge poses additional problems. The last problem is that there is no gold standard for in vivo tractography.

As fibre tracking requires more extensive computer calculations and manpower than DWI or the more complex DTI it remains more of a research instrument and has, to date, no or very limited clinical application. However, it seems most likely that diffusion tensor MRI fibre tractography will improve our understanding of brain pathology, especially that of white matter abnormalities. Possible future applications include the evaluation of these parameters with closed head injury and stroke, and even the evaluation of peripheral nerve injuries. Fibre tracking can be used in conjunction with functional MRI (fMRI) to analyse the anatomical connections and functional pathways for diagnostic purpose and in pre-surgical planning (26).

\section{Clinical applications}

While DWI-MRI has several clinical applications and is routinely used in stroke work-up, DTI is still used primarily in research and not as routine examination at most institutions. Increasing number of reports is trying to demonstrate the value of DTI as a tool for evaluation of different entities in the brain. The following text reviews some of the clinical areas in which DTI has shown to be of interest.

\section{$D T I$ in cerebral ischaemia, leucoaraiosis and wallerian degeneration}

With cerebral ischaemia, a decrease in cerebral blood flow below $10-15 \mathrm{ml} / 100 \mathrm{~g} / \mathrm{min}$ leads to increased volume of intracellular water. This influx of water from the extracellular compartment causes the cells to swell, which manifests as cytotoxic oedema. With conventional MR technique, the acute stage of ischaemia is poorly assessed and the extent of the ischaemic parenchymal tissue can only be demonstrated at a later stage when the vasogenic oedema is present. DWI and DTI have been extensively used to reliably detect hyperacute ischaemic brain injury while the conventional MRI is still normal (1, 29-31). Both DWI and DTI also make it possible to distinguish the acute from the chronic in the course of ischaemic changes, which may have impact on treatment. In the acute phase, ADG is initially reduced by $30-50 \%$ within 30 min after the onset of focal ischaemia (29-32), and is more severely reduced in the white matter compared to the grey matter during acute and early subacute infarcts $(33,34)$. With ischaemic brain trauma, DTI parameters, such as the $D_{\mathrm{av}}$ (equivalent to ADC), initially decrease but subsequently increase and become higher than normal values. In fact, these values remain elevated in the chronic phase of injury (35). In a short interval between decreased $\mathrm{ADC}$ values and the subsequently increased $\mathrm{ADC}$ values there is a short time period during which the values are normal, a process referred to as pseudonormalization. Studies have indicated that this pseudonormalization occurs approximately 9 days after a cerebral stroke in adults, and in newborns, approximately 7 days after injury (35). In addition to the changes in the ADC values, an acute elevation in FA has been observed in ischaemic white matter but not in ischaemic grey matter (36). This acute elevation is followed by a marked reduction in FA during the chronic phase (36).

These described changes are believed to be due to loss of organization in normal structures when their cytoarchitecture is disrupted.

In contrast to the re-normalization and subsequent elevation of the ADC values seen in chronic stroke, the diffusion anisotropy remains significantly reduced in the infarcted area compared to the homologous contralateral region of the brain, even 2-6 months after ischaemic stroke (37). It has been demonstrated that ADC threshold values are useful in predicting tissue viability and stroke outcome $(33,38)$ and it has also been shown that by combining ADC and anisotropy data it is possible to evaluate strokes and predict clinical outcome (33).

In this context it has to be born in mind that there are clinical conditions under which the ADC increases rather than decreases after an injury. In general, it appears that ADC decreases immediately after cell injury in the presence of cytotoxic oedema. However it may be increased early on under conditions of vasogenic oedema, for example, in the reversible posterior leucoencephalopathy syndrome $(13,14)$, or in high-pressure hydrocephalus (39).

Leucoaraiosis is a non-specific name describing the radiological appearance of diffuse changes in the periventricular white matter seen either on CT or MR images (40).

Leucoaraiosis appearance may be seen in various diseases including chronic ischaemia, Alzheimer's disease, and CADASIL (cerebral autosomal dominant arteriopathy with subcortical infarcts and leucoencephalopathy); histopathological findings of axonal loss and proliferation of glial cells have been reported in leucoaraiosis $(41,42)$. In patients with ischaemic leucoaraiosis, in areas of increased signal on T2-weigthed images, DTI showed elevated mean diffusivity and reduced FA (41). Significant changes in FA in normal appearing white matter in patients with vascular dementia have been demonstrated and these changes correlated with executive dysfunction (43). In addition, the mean diffusivity observed in leucoaraiosis has shown to be significantly lower than that observed in a lacunar infarct, most likely due to proliferation of glial tissue in leucoaraiosis, which interferes with water diffusion (44).

Wallerian degeneration (WD) is described as antegrade degeneration of axons and their myelin sheaths secondary to proximal axonal injury or cell death, and is most commonly involving the corticospinal tracts, secondary to ipsilateral cerebral infarction. 
It has been shown that DTI is more sensitive than T2weighted imaging in detecting WD (45). That investigation demonstrated that diffusion anisotropy is reduced both in the primary lesion and in the areas of WD, whereas the ADC is only slightly increased in WD but is markedly increased in primary stroke lesions (45). This means that ADC may have the potential to distinguish primary stroke lesions from areas of WD (45).

\section{$D T I$ in the developing brain, brain maturation and ageing}

Several challenges exist for the application of DTI in the developing human brain. The value for the water ADC and diffusion anisotropy differs markedly between the paediatric and the adult brain and both values also vary with age (21-23). However, similar MR pulse sequences and post-processing methods are used for both infant and adult human brain DTI with the exception of the $b$-value which is typically chosen in the order of $1000 \mathrm{~mm}^{2} / \mathrm{s}$ for adults and in the order of $700-800 \mathrm{~mm}^{2} / \mathrm{s}$ for infants.

Significantly higher ADC and lower FA in neonates have been found compared with those in adults (46). In addition, the ADG value has been shown to be higher in the white matter compared with the grey matter in the paediatric brain $(21,22)$. The ADC values for the white matter of the centrum semiovale in premature infants approach $2.0 \times 10^{-3} \mathrm{~mm}^{2} / \mathrm{s}$. Thereafter, with increasing age, the ADC values decrease and the anisotropy values especially the RA - increase in a monotonic fashion during development until they reach the ADC values of the adult brain (which typically are $\left.0.7 \times 10^{-3} \mathrm{~mm}^{2} / \mathrm{s}\right)(21,22)$. The changes in ADC values predominantly occur within the first 6 months of life and are believed to be related to decreased total water content, myelination, and the organization of the white matter tracts, which all decrease the diffusivity (47-49). This value, in turn, changes with increasing age and when measured in different parts of the brain $(46,50)$. Lower FA values have been found in the frontal white matter in children compared to values found in young adults suggesting that the measured diffusion anisotropy is directly related to the maturation of white matter (51).

Recently, DTI has been used in the evaluation of normal ageing to detect age-related degeneration $(23,52)$. Previous work has shown an age-related ADC elevation in the cerebral white matter of adults older than 40 years of age compared with that of younger individuals (53). This decrease has been shown to occur already after 20 years of age. A previous study looking at normal ageing found that diffusion anisotropy, a marker of white matter tract integrity was reduced in the white matter in older individuals and fell linearly with increasing age, whereas the mean diffusitivity was higher in older individuals and increased with increasing age (54). That study also found that the increase in anterior mean diffusitivity in the elderly individuals correlated with executive function. Significant age-related declines in median FA have been demonstrated in the anatomically determined areas of densely packed white matter fibres, especially in genu of corpus callosum and centrum semiovale (23).

These findings of age-related decrease in white matter anisotropy suggest that white matter tract disruption occurs in normal ageing and would be consistent with the cortical disconnection hypothesis of age-related cognitive decline, and that it has to be taken into account when evaluating effects of different diseases, especially in the elderly. An abnormal decline in FA can be exemplified by a recent study which showed that, relative to age-matched control subjects, alcoholic men have lower regional FA values in the genu of the corpus callosum and in the centrum semiovale (55).

Another study also demonstrated regional abnormalities in white matter microstructure, not detectable on conventional MRI in patients with chronic alcoholism (56), supporting the theory that alcoholism disrupts white matter microstructure, which contributes to deficits in attention and working memory.

\section{$D T I$ and diffuse axonal injury}

Focal brain injuries other than stroke have not yet been widely studied with DTI, and it is not fully known whether mechanisms similar to those reported in ischaemic stroke are involved in their recovery. The entire mechanisms underlying the changes in diffusion anisotropy are not yet fully understood but it has been suggested that they probably reflect changes in the underlying microscopic structure of the examined tissue. In ordered structures like white matter tracts, a loss of order or structural integrity of the tissue results in a reduction of the FA $(33,57)$. The diffusion along axons decreases, while the diffusion in directions perpendicular increases (58). The traumatic brain injuries can be classified in two main categories: focal and diffuse brain injury. The focal brain injury, such as haematoma and cerebral contusion, results from direct impact trauma to the head whereas diffuse brain injury results from shearing injuries and tensile strain on the brain as a result of abnormal rotation or deceleration of adjacent tissues that differ in density or rigidity. The resulting tissue injury is characterized by axonal stretching, disruption, and eventual separation of nerve fibres $(59,60)$. It has been shown that DWI is able to detect additional shearing injuries not visible on T2 or FLAIR images but also that it is less sensitive than $\mathrm{T} 2 *$ imaging in detecting hemorrhagic lesions (61). In that study ADC maps were performed in all patients and the majority $(65 \%)$ of the DWI positive lesions showed decreased diffusion (61). The reduced ADC after traumatic axonal shearing injury can persist much longer than is observed with large vessel territorial ischaemia (62). Surrounding a focal brain lesion such as a contusion or a focal haematoma, there is zone of apparently 'tissue at risk' defined by reduced diffusivity. This apparent zone might 
be of importance in future treatment and in the development of new therapies to save this zone in patients with head trauma (63). Most of the histopathological abnormalities seen in diffusion anisotropy values appear to be in the internal capsule and in the corpus callosum and less in the grey matter such as thalamus and putamen. A recent retrospective study of 20 patients with diffuse axonal injury demonstrated a significant reduction of ADC values within the splenium of the corpus callosum and significant reduction of FA values in the internal capsule and splenium of the corpus callosum (58) (Fig. 2). That study also showed that the correlation between the FA-values and clinical makers, i.e. GCS/Rankin scores was better than the correlation between $\mathrm{ADC}$ values and clinical markers,

(a)

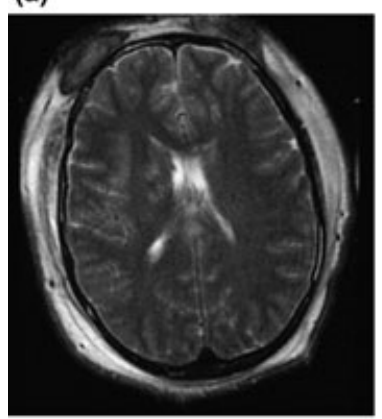

(c)

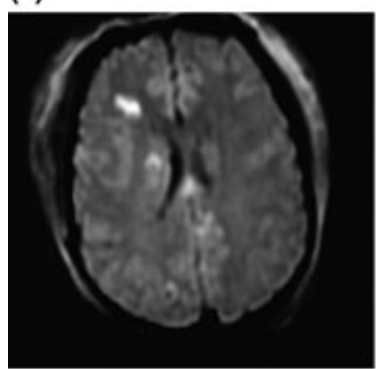

(e)

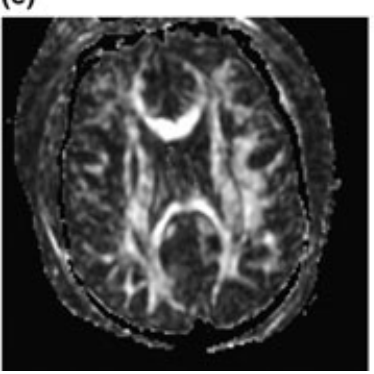

Fig. 2. Axonal brain injury. A 18-year-old male who was an unrestrained passenger in a motor vehicle accident. Small focal areas of slightly increased signal, present in the white matter of the right frontal lobe and corpus callosum on T2-weighted (a) and flair (b) images. The diffusion-weighted images (DWI) demonstrate scattered areas of increased signal within the right frontal lobe and in the splenium and body of the corpus callosum as can be seen in axonal shearing injury and ischaemia (c). The ADC maps show focal areas of decreased ADC (d) and decreased FA is seen on the FA map in the same region (e). suggesting that FA changes may be able to serve as predictive parameters for clinical outcome (58).

It has been noted that the significant reduction of diffusion anisotropy seen in normal appearing white matter on conventional MRI within the first $24 \mathrm{~h}$ after diffuse axonal injury is less evident, even though still abnormal, weeks after the trauma (57). A decrease in the diffusion coefficient has been demonstrated in normal or almost normal appearing areas on T1, T2 and FLAIR images already within the first day of life in newborns with high risk of perinatal brain injury. However, this decrease in diffusion coefficient became more obvious at third day of life with a normalization of the values within a week - so-called pseudonormalization (64). At this point in time the conventional MRI was abnormal. These findings suggest that DTI obtained on the first day after an injury might not show the full extent of the injury in newborn infant and that images preformed 3 days after the injury might be better predictors of the final extent of the injury (64).

Noteworthy is that many of previous studies are small and limited, and therefore, it is obvious that further research with larger longitudinal studies and correlation with pathological specimens and clinical outcome is needed to fully evaluate the use DTI in the estimation of the full extent of a brain injury, in the prediction of clinical outcome, and to find out the optimal timing for the MRI examination.

\section{$D T I$ in the evaluation of brain tumour}

Despite the information provided by conventional MR with contrast-enhanced T1- and T2-weighted sequences to characterize the location and extent of the tumour the radiological specification and grading of a brain tumour is still limited. Therefore there is a growing interest in the use DWI and DTI to evaluate different tumour components, and to differentiate tumour invasion from normal brain tissue or oedema. ADC maps have proved to be helpful in defining solid enhancing tumour, no contrast enhancing lesion, peritumoural oedema, and necrotic and/or cystic regions from normal surrounding brain tissue $(11,12,65-$ 70).

It has been demonstrated that cystic or necrotic regions have highest ADC values (65) while contrast-enhancing part of the tumour has lower ADC than both the cystic or the necrotic areas and oedema $(11,12,65-70)$. A recent study has shown that FA values and histograms of mean diffusivity in peritumoural areas could not be used to differentiate high-grade from low-grade gliomas or meningiomas from intraaxial tumours (71). Other recent studies have demonstrated similar findings with a trend towards reduction of FA but not of mean diffusitivity in the peritumoural oedema of glioblastomas compared to meningioma (72) and also alterations in FA in white matter in the affected as well as in the contralateral hemispheres in 
glioblastomas but not in metastases (73). However, significant increase in mean diffusivity and significant decrease in FA have been demonstrated in the peritumoural region of both gliomas and metastatic tumours when compared with those of normal appearing white matter (74). Furthermore, the peritumoural mean diffusivity of metastatic lesions measured significantly greater than that of gliomas whereas the FA values showed no discrepancy between tumour and metastatic lesions, suggesting that the FA changes in areas surrounding gliomas can be attributed to both increased water content and tumour infiltration (74). Such infiltration may result in deviation and disorganization in the white matter as has been seen in tractography studies $(75,76)$.

Other measurements such as Tumour Infiltration Index (TII) have recently been introduced to separate tumours with presumed purely vasogenic oedema, i.e. metastases and extra-axial lesions such as meningioma from those with tumour-infiltrated oedema, i.e. high- and low-grade glial tumours. The mean TII was significantly higher in presumed tumour-infiltrated oedema (71).

DWI and DTI both in the adult as well as in the paediatric population have also been used to explore the possibility of differentiating the type and the grade of a tumour. Several studies have shown that low-grade astr- ocytoma has higher ADC values whereas high-grade malignant glioma has lower ADC values - findings reflecting more restricted diffusion with increasing tumour cellularity $(12,67,68,77)$. However a recent study concluded that differentiation of tumours by the use of FA values which are generally reduced in tumours suggesting structural disorder does not add any additional information to the tissue differentiation (69), but may help in the understanding of the effect of brain tumours on white matter fibres, which may be important in the presurgical planning $(76,78)$. In a small DTI study four major patterns have been identified in the affected white matter tracts, categorized on the basis of anisotropy and fibre direction or orientation. Such patterns may potentially be helpful in pre-surgical planning (79) (Fig. 3).

In the treatment of patients with tumour it is crucial to obtain reliable information about tumour response to therapy. Recent data from both animal models and human studies suggest that diffusion imaging may be a sensitive tool in the evaluation of tumour response to therapy (80-82) and be helpful in predicting chemosensitivity of glial tumours (83). Early increasing ADG values during therapy may relate to therapy-induced cell necrosis. The subsequent drop in ADC values within the tumour to pretreatment levels is thought to be an indicator of tumour (a)

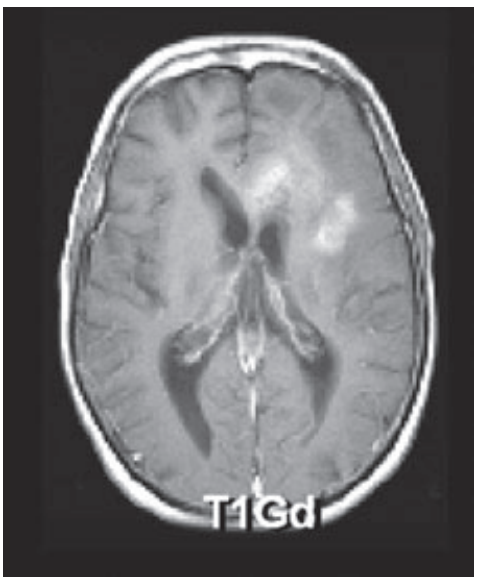

(c)

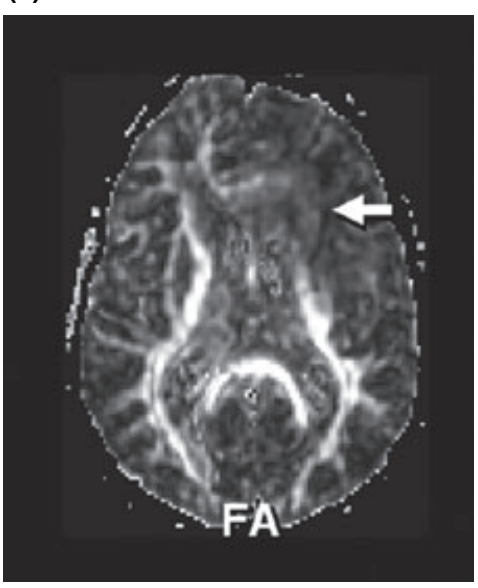

(b)

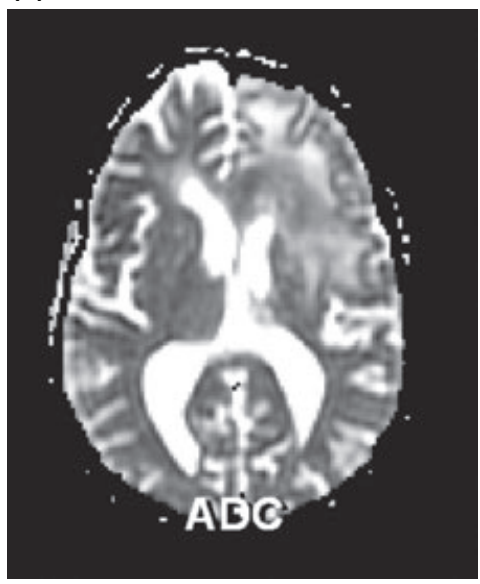

(d)

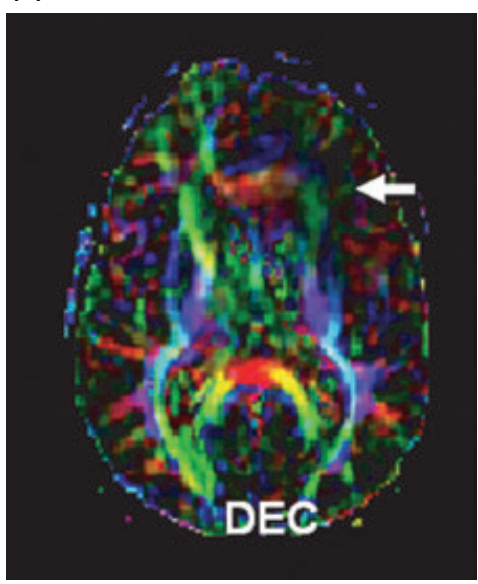

Fig. 3. Brain tumour. A 63-year-old woman with a left frontal astrocytoma. T1-weighted post-contrast enhanced images demonstrates pathological contrast enhancement in the left frontal lobe and the genu of the corpus callosum (a). The apparent diffusion coefficient (ADC) was slightly increased compared to normal regions of the brain (b) and the fractional anisotropy (FA) value was decreased (c). The disruption of the white matter tracts can nicely be seen on the directional encoded images (DEC) (d). 
re-growth. These findings are in part in contradiction to results that demonstrate increased $\mathrm{ADC}$ values in recurrent tumours compared to those obtained in patients with radiation injury/necrosis.

A recent study demonstrated the possibility to use ADCmaps and relative cerebral blood volume measurements to evaluate chemosensitivity; areas of the tumour with the lowest ADC values and highest rCBV demonstrated a significant volume reduction during treatment whereas other components of the tumour demonstrated no change in volume (83).

\section{$D T I$ in the evaluation of radiation injury versus recurrent tumour}

Enhancing lesions that arise on routine follow-up brain MRI at the site of a previously identified and treated primary intracranial neoplasm may present a significant diagnostic dilemma. These lesions are typically subjected to radiation and/or chemotherapy and in most instances surgical resection. Many do not have specific imaging characteristics that enable the neuroradiologist to discriminate tumour recurrence from the inflammatory or necrotic changes which result from treatment with radiation and/or chemotherapy (84). Both recurrent tumours and treatment-related changes (i.e. necrosis) typically demonstrate enhancement with gadolinium and are commonly surrounded by an area of increased T2 signal. However, recent reports have suggested that diffusion measurements can potentially provide important diagnostic information regarding newly enhancing lesions as well as of the surrounding areas of signal abnormality that may appear months to years after radiation therapy in patients previously treated for primary and metastatic brain tumours $(85,86)$. We found in a recent study of 19 patients who presented with a new area of contrast enhancement at, or nearby, the site of previously treated (surgery/biopsy and radiation therapy ) neoplasm that the ADC values were significantly higher in patients with tumour recurrence (mean $1.23 \times 10^{-3} \mathrm{~mm}^{2} / \mathrm{s}$ ) than in those with treatment related changes (mean $1.07 \times 10^{-3} \mathrm{~mm}^{2} / \mathrm{s}$ ) (Figs 4 and 5). FA values were lower in both groups compared with FA values in normal appearing frontal white matter but no statistical difference was seen in the FA values in the contrast-enhancing lesion between the two groups (85, unpublished data). Increased ADC values in recurrent tumours have also been reported by others (87-89). Contradictions in literature exist regarding the question whether an increased $\mathrm{ADC}$ is to be expected in tumour or radiation injury/necrosis. In a recent study the recurrence group showed statistically lower mean ADC values (mean $1.18 \times 10^{-3} \mathrm{~mm}^{2} / \mathrm{s}$ ) compared with the treatment-induced necrosis group (mean $1.40 \times$ $10^{-3} \mathrm{~mm}^{2} / \mathrm{s}$ ) (86). Also, in another study higher ADC values were seen in treatment-related changes/radiation necrosis than in solid tumours (90).
Two recent studies have shown that although the mean ADC values in areas of T2-prolongation, surrounding the contrast enhancing lesion differ compared to values obtained in normal brain tissue no significant differences are demonstrated between patients with radiation injury and those with recurrent tumour $(86,91)$. One of these studies demonstrated significantly higher ADC ratios (ADC in abnormal area/ADC in normal appearing brain tissue) in the oedema in patients with radiation injury compared to those with tumour recurrence (91). More interestingly, the same study demonstrated that for the normal appearing white matter tract adjacent to the oedema, both the FA values and the FA ratios were significantly lower in patients with recurrent tumour compared to those with radiation injury. These findings may indicate that the DTI provides a helpful method in detecting occult white matter tract invasion and the FA measurement could be more sensitive than ADC.

Noteworthy limitations for all these studies are the small number of patients and the lack of histological confirmation in all cases. There is certainly a need for further exploration with larger studies, and correlation with histopathological specimens and clinical outcome in this field.

\section{DTI and multiple sclerosis}

Numerous studies have been published on MRI diffusion measurements in MS. All studies have shown that the mean diffusivity is elevated in lesions that are seen on T2weighted images. The degree of elevation seems to be somehow related to the clinical course of the disease. The lesions with more 'destructive' pathology are generally shown to have the most strongly elevated diffusivity $(7,52$, 92-94). An elevated diffusivity (4-13\%) has also been found in normally appearing white matter of MS patients this interesting fact may suggest that MS is a diffuse white matter disease as well as multifocal in nature. On the other hand those findings also may suggest that the white matter may be subjected to WD proximal and distal to the visible lesions (7, 8, 93-95).

In addition, a recent study has demonstrated increased water diffusivity in the cerebral grey matter of MS patients, suggesting that the grey matter may not be spared by the pathological process (96). A recent study assessing the magnitude of cognitive impairment in patients with relapsing-remitting MS concluded that moderate correlation of mean ADC and mean FA values seems to reflect the severity of language, attention, and memory deficits in these patients $(97)$.

It appears that the FA values are more sensitive than $\mathrm{ADC}$ values in detecting white matter abnormalities in MS (93, 98, 99). A few studies have demonstrated generally reduced diffusion anisotropy in MS lesions, in one study the lowest anisotropy was seen in acute inflammatory lesions (93) while another study found the lowest anisotropy in T-1 hypointense lesions (94) (Fig. 6). 
(a)

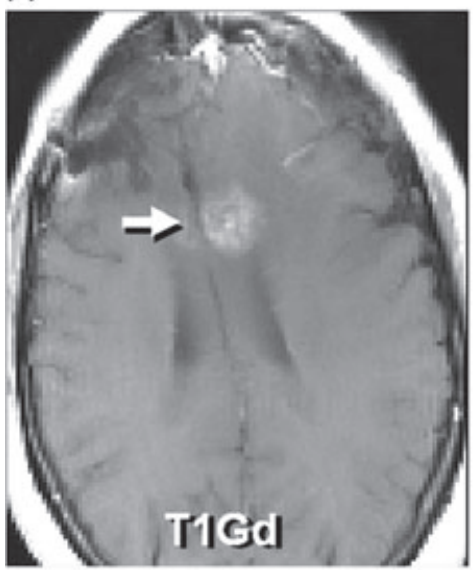

(c)

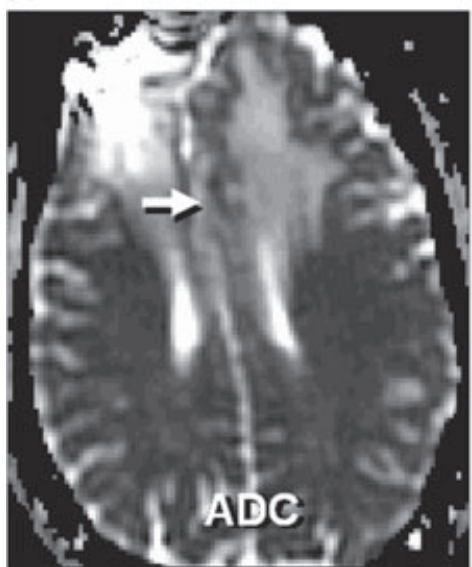

(e)

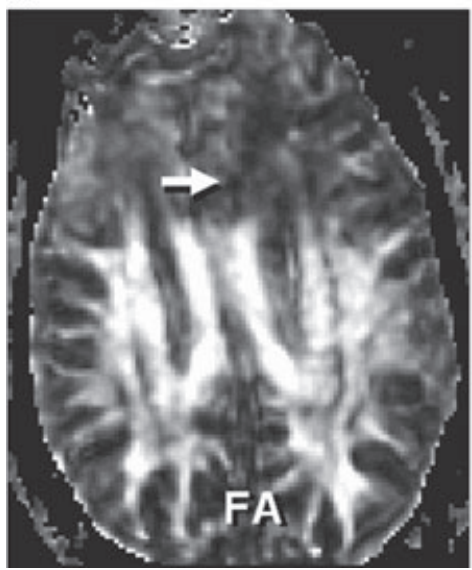

(b)

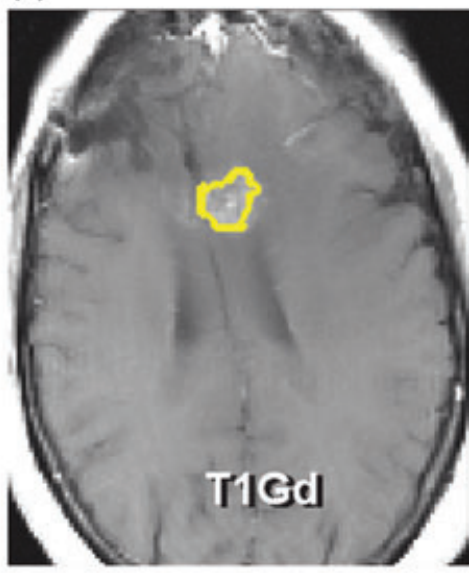

(d)

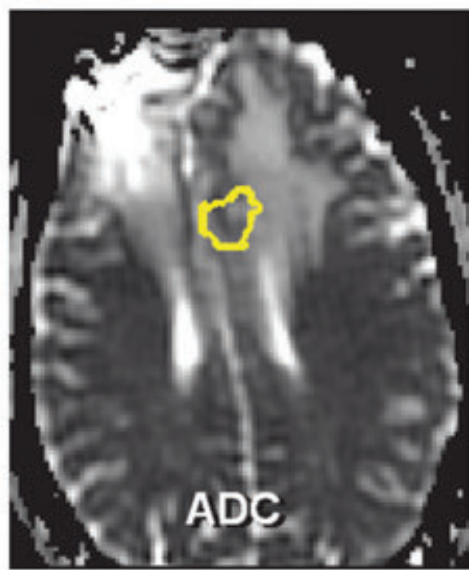

(f)

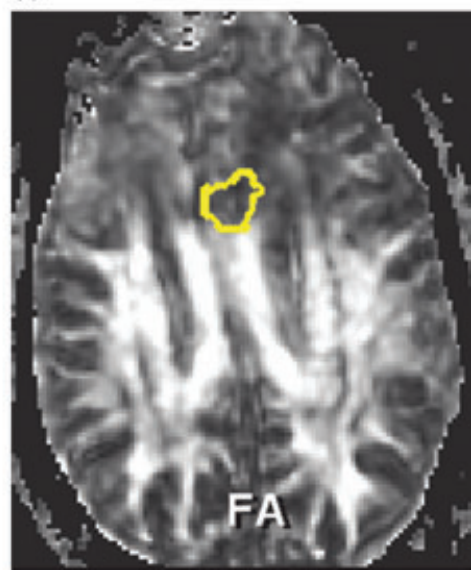

Fig. 4. Radiation injury. A 42-year-old woman, status post surgery and radiation for an anaplastic oligoastrocytoma with new contrast enhancing lesions in the left frontal lobe and left aspect of the corpus callosum, as seen on the T1-weighted post-contrast enhanced image (a). The patient was evaluated for radiation injury versus recurrent tumour and an ROI was placed over the contrast-enhancing lesion (b). ADC maps (c, d) and FA-maps $(\mathbf{e}, \mathbf{f})$ were generated. The ADC value of the solid lesion was $1.04 \times 10^{-5} \mathrm{~mm}^{2} / \mathrm{s}$ and the FA ratio of the solid lesion was 0.28 .

\section{$D T I$ and epilepsy}

Hippocampal sclerosis is a common cause of refractory complex partial seizures from the temporal lobes (100). High resolution MRI has been used in identifying specific features such as hippocampal volume loss and T2 or
FLAIR signal hyperintensity (101, 102). Recent studies utilizing DTI in patients with chronic epilepsy and hippocampal sclerosis have demonstrated increased diffusivity and reduced anisotropy in sclerotic hippocampi, which are findings suggesting loss of structural organization and expansion of the extracellular space (103, 104). These 
(a)

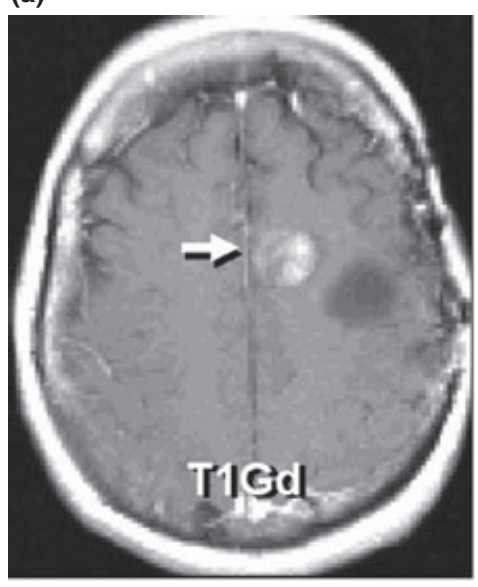

(c)

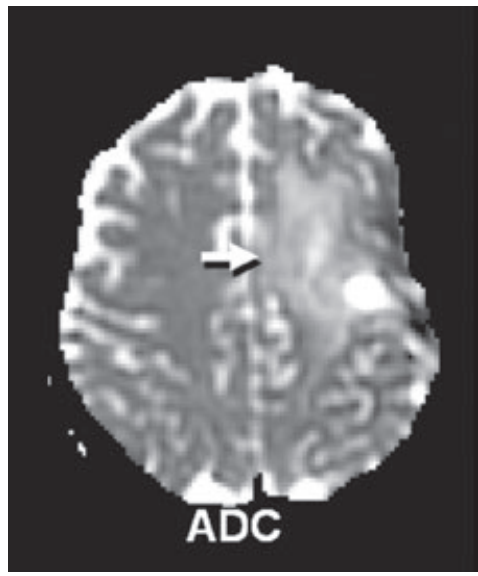

(e)

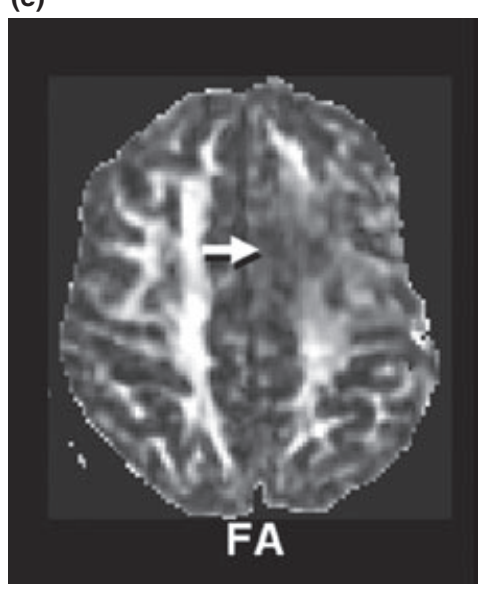

(b)

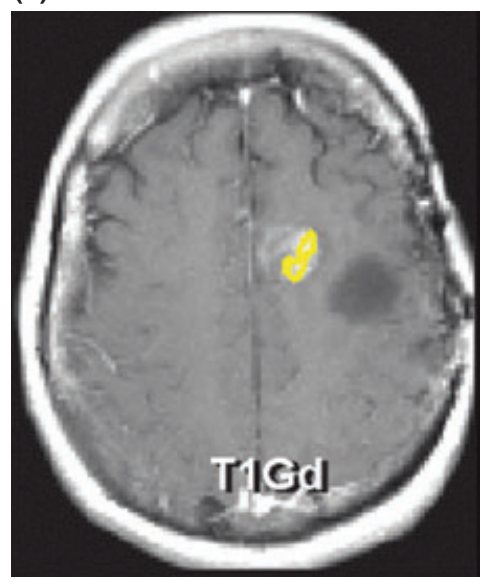

(d)

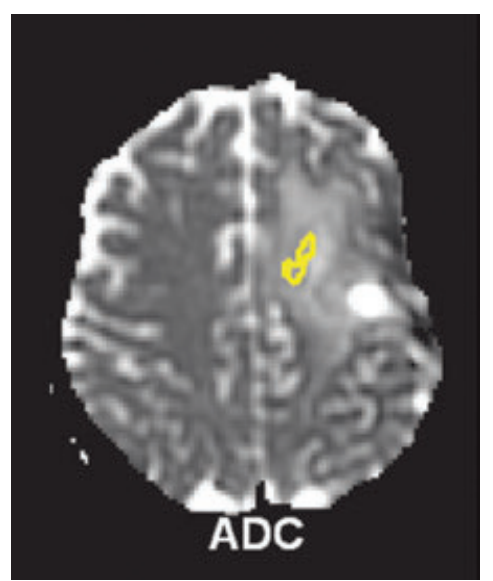

(f)

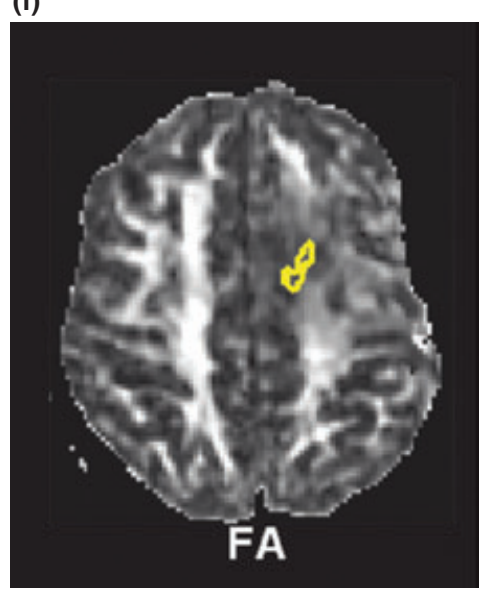

Fig. 5. Recurrent tumour. A 37-year-old woman status post-surgery, chemo- and radiation therapy for left frontal astrocytoma, presenting with new contrast enhancing lesion in the left frontal lobe, as seen on T1-weighted post-contrast enhanced images (a). The patient was evaluated for radiation injury versus recurrent tumour and an ROI was placed over the contrast-enhancing lesion (b). ADC maps $(\mathbf{c}, \mathbf{d})$ and FA maps $(\mathbf{e}, \mathbf{f})$ were generated. The ADC value of the solid lesion was $1.37 \times 10^{-5} \mathrm{~mm}^{2} / \mathrm{s}$ and the FA ratio of the solid lesion was 0.38 .

changes in diffusivity have shown to extend beyond the origin of seizure, involving normally appearing brain tissue (104). There are patients with refractory epilepsy and extra-temporal, neocortical epilepsy, in which high-resolution MRI techniques have identified malformations of cortical development (MCD) as potential epileptogenic foci. Pre- liminary data in 12 patients with refractory epilepsy and cortical signal abnormalities showed differences in the ADG values within the lesion compared to normal brain tissue in the same location on the contralateral side, whereas there was a significant overlap between the FA values (105). DTI was utilized in another investigation to 
(a)

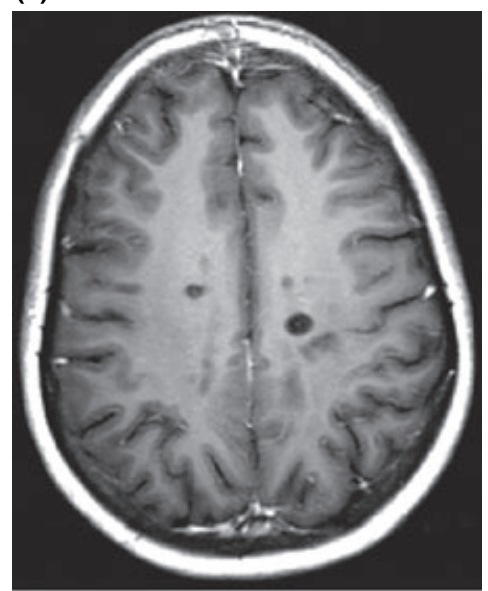

(c)

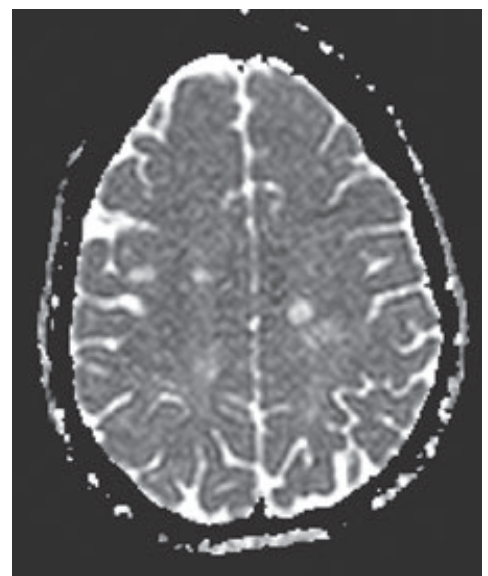

(b)

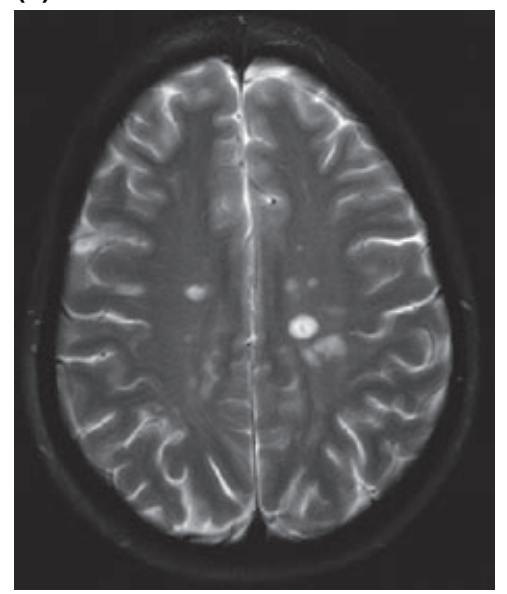

(d)

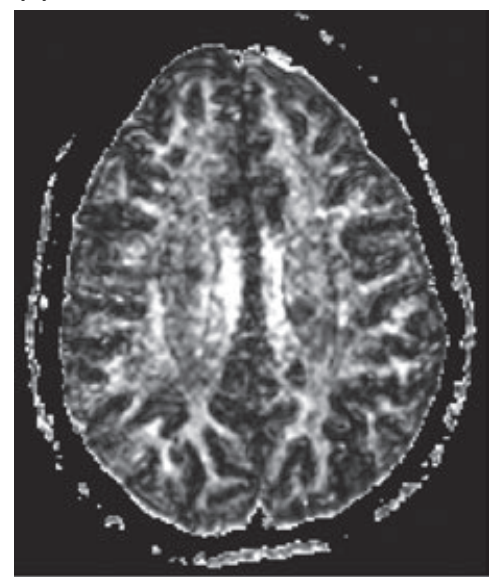

Fig. 6. Multiple sclerosis. A 43-year-old woman with multiple sclerosis. T2-weighted image demonstrates multiple areas of increased signal representing plaques (a). T1-weighted post-contrast enhanced image (b); none of them demonstrate any pathological contrast enhancement. These lesions demonstrate increased ADC (c) and decreased (d) FA values.

demonstrate higher diffusivity and lower anisotropy not only in the area of MCD but also in surrounding brain tissue that appeared normal on conventional MRI (106). Also others have reported similar abnormalities in diffusivity and anisotropy in normally appearing brain tissue beyond the origin of seizure $(107,108)$.

A recent study used DTI to study bifrontal schizencephaly and subcortical heterotopia, showing low FA within the subcortical heterotopia, including that lining the cleft. The study also demonstrated deviation in the direction of the corticospinal tracts surrounding the heterotopic grey matter as compared to normal subjects and by using fibre tracking the authors could demonstrate the corticospinal tracts to be smaller and widely separated from the pyramidal fibres, and also an alteration in white matter fascicles (109). Other authors have used DTI to study white matter pathways in band heterotopia (105) and holoprosencephaly (110).

It is evident that DTI may prove invaluable in identifying potential epileptogenic foci, in describing aberrations of white matter fibre tracts as well as in more precise defining of the extent of lesion for surgical resection.

\section{DTI and cognitive decline and Alzheimer's disease}

Studies of Alzheimer's disease (AD) have concentrated on changes in anisotropy associated with $\mathrm{AD}$ progression. Changes in diffusion anisotropy have been demonstrated by measuring diffusivity in the corpus callosum at the midline where axons are primarily oriented in the leftright direction (111). That study showed that the anisotropy was decreased in the genu and the splenium of the corpus callosum in AD patients compared with that of sex- and age-matched controls, most likely due to axonal loss or demyelination in these areas (111). In another study the integrity of axonal tracts in areas associated with cognitive function was compared with this of tracts associated with motor function (112). By using the lattice index (20) as a measure of white matter tract integrity, the author's demonstrated preservation of the motor tracts but axonal degeneration in the cognitive tracts (112). 
DTI has also been used in the evaluation of different forms of cognitive impairment in patients other than those with Alzheimer's disease, such as in adults with reading difficulties (113), and in detecting degenerated fibre tracts in the disconnection syndrome (114). A recent investigation showed decreased diffusion anisotropy bilaterally in the temporo-parietal white matter in subjects with reading difficulty (113). That study demonstrated that the white matter diffusion anisotropy in the temporo-parietal region of the left hemisphere was significantly correlated with reading scores within the reading-impaired adults and within the control group, reflecting the microstructure of white matter tracts, which may contribute to reading ability (113).

\section{Metabolic disorders}

Lately, several reports on DTI in the diagnosis of metabolic disorders, such as malignant phenylketonuria (PKU) (115), adrenoleucodystrophy (ALD) (116-118), and Krabbe's disease (119), and in the differentiation of dysmyelinating from the demyelinating disorders $(120,121)$ have been published.

A recent study, in which patients with treated chronic malignant PKU were evaluated, demonstrated significantly lower FA values in the parieto-occipital and central white matter in PKU patients older than 3 years and also the eigenvectors (EV) EV2 and EV3 were significantly larger than those in the controls, and the findings were, in part, correlated with IQ (115). In X-linked ALD a decrease in FA and an increase in ADC have been demonstrated (117, 118): these changes in FA values and ADC within affected white matter correlate with previously described histopathological zones seen in this disease (122). These findings suggest an increase in free water and the loss of the integrity of the myelin sheaths and axons in the white matter, resulting in increased diffusivity.

Finally, DTI has been found to be superior to conventional MRI in differentiating dysmyelinating disorders, such as Pelizaeus-Merzbacher disease from demyelinating disorders, such as Krabbe's disease and Alexander's disease. Diffusional anisotropy remains present in dysmyelinated lesions but is lost in demyelinated lesions - findings verified both in animal and human studies $(120,121)$.

\section{Conclusion}

The recent development of DTI allows direct examination, in vivo, of some aspects of the brain tissue microstructure. The use of DTI has already shown to be valuable in neuroanatomy, fibre connectivity and in examination of brain development. It has become a highly interesting tool in the evaluation of different pathological processes in the brain, such as cerebral ischaemia, traumatic brain injury, multiple sclerosis, Alzheimer's disease and cognitive impairment, epilepsy, brain tumours and metabolic disorders.
However, further improvements in technique and stable post-processing analyses are needed. There is significant increase in the utilization of DTI both in research and in clinical applications.

\section{Acknowledgements}

I wish to express a special thank you to Professor Thomas L Chenevert PhD, Xiaoying Fan MD and Robert C Welsh $\mathrm{PhD}$ for their valuable comments and support and for their kind help with image preparations.

\section{References}

1. Moseley M, Cohen Y, Kucharczyk J, et al. Diffusion-weighted MRimaging of anisotropic water diffusion in cat central nervous system. Radiology 1990; 176: 439-445.

2. Le Bihan D. Molecular diffusion nuclear magnetic resonance imaging. Magn Reson Q 1991; 7: 1-30.

3. Bammer R. Basic principles of diffusion-weighted imaging. EJR 2003; 45: 169-184.

4. Moseley ME, Kucharczyk J, Mintorovitch J, et al. Diffusion-weighted MR imaging of acute stroke: correlation with T2-weighted and magnetic susceptibility-enhanced MR imaging in cats. AJNR Am J Neuroradiol 1990; 11: 423-429.

5. Lansberg MG, Norbash AM, Marks MP, Tong DC, Moseley ME, Albers GW. Advantages of adding diffusion-weighted magnetic resonance imaging to conventional magnetic resonance imaging for evaluating acute stroke. Arch Neurol 2000; 57: 1311-1316.

6. Larsson HBW, Thomsen C, Fredriksen J, Stubgaard M, Henriksen O. In vivo magnetic resonance diffusion measurements in the brain of patients with multiple sclerosis. Magn Reson Imaging 1992; 10: $7-12$.

7. Horsfield MA, Lai M, Webb S, et al. Apparent diffusion coefficients in benign and secondary progressive multiple sclerosis by nuclear magnetic resonance. Magn Reson Med 1996; 36: 393-400.

8. Christensen P, Gideon P, Thomsen C, Stubgaard M, Henriksen O, Larsson HBW. Increased water self-diffusion in chronic plaques and in apparently normal white matter in patients with multiple sclerosis. Acta Neurol Scand 1993; 87: 195-197.

9. Nakahara M, Ericsson K, Bellander BM. Diffusion-weighted MR and apparent diffusion coefficient in the evaluation of severe brain injury. Acta Radiol 2001; 42: 365-369.

10. Sundgren PC, Reinstrup P, Romner B, Holtas S, Maly P. Value of conventional, and diffusion-and perfusion weighted MRI in the management of patients with unclear cerebral pathology, admitted to the intensive care unit. Neuroradiology 2002; 44: 674-680.

11. Stadnik T, Chaskis C, Michotte A, et al. Diffusion-weighted MR imaging of intracerebral masses: comparison with conventional MR imaging and histologic findings. AJNR Am J Neuroradiol 2001; 22: 969-976.

12. Kono K, Inoue $\mathrm{Y}$, Nakayama $\mathrm{K}$, et al. The role of diffusionweighted imaging in patients with brain tumors. AJNR Am J Neuroradiol 2001; 22: 1081-1088.

13. Sundgren PC, Edvardssson B, Holtas S. Serial investigation of perfusion disturbances and vasogenic oedema in hypertensive encephalopathy by diffusion and perfusion weighted imaging. Neuroradiology 2002; 44: 299-304.

14. Hinchey J, Chaves C, Applgnani B, et al. A reversible posterior leukoencephalopathy syndrome. N Engl J Med 1996; 334: 494-500.

15. Le Bihan D. Clinical Perfusion and Diffusion Imaging. Raven Press, New York, 1993.

16. Le Bihan D, Mangin JF, Poupon C, Clark CA, Pappata S, Molko N. Diffusion Tensor Imaging: Concepts and Applications. Magn Reson Imaging 2001; 13: 534-546. 
17. Pipe JG, Farthing VG, Forbes KP. Multishot diffusion FSE using PROPELLER MRI. Magn Reson Med 2002; 47: 42-52.

18. Bammer R, Auer M, Keeling SL, et al. Diffusion tensor imaging using single shot SENSE-EPI. Magn Reson Med 2002; 48: 123-136.

19. Basser PJ, Jones DK. Diffusion tensor MRI: theory, experimental design and data analysis - a technical review. NMR Biomed 2002; 14: 456-467.

20. Pierpaoli G, Basser PJ. Toward a quantitative measurement of diffusion anisotropy. Magn Reson Med 1996; 36: 893-906.

21. Mukherjee P, Miller JH, Shimony JS, et al. Normal brain maturation during childhood: developmental trends characterized with diffusion-tensor MR imaging. Radiology 2001; 221: 349-358.

22. Morris MC, Zimmerman RA, Bilanluk LT, Hunter JV, Hasselgrove JC. Changes in brain water diffusion during childhood. Neuroradiology 1999; 41: 929-934.

23. Pfefferbaum A, Sullivan EV, Hedehus M, Lim KO, Adalsteinsson E, Moseley M. Age-related decline in brain white matter anisotropy measured with spatially corrected echo-planar diffusion tensor imaging. Magn Reson Med 2000; 44: 259-268.

24. Pajevic S, Pierpaoli C. Color schemes to represent the orientation of anisotropic tissues from diffusion tensor data; application to white matter fiber tract mapping in the human brain. Magn Reson Med 1999; 42: 526-540.

25. Mori S, Van Zijl PC. Fiber tracking: principles and strategies - a technical review. NMR Biomed 2002; 15: 468-480.

26. Lori NF, Akbudak E, Shimony JS, et al. Diffusion tensor fiber tracking of human brain connectivity: acquisition methods, reliability analysis and biological results. NMR Biomed 2002; 15: 493-515.

27. Basser PJ, Pajevic S, Pierpaoli C, Duda J, Aldroubi A. In vivo fiber tractography using DT-MRI data. Magn Reson Med 2000; 44: 625632 .

28. Bammer R, Acar B, Moseley M. In vivo MR tractography using diffusion imaging. Eur J Radiol 2002; 45: 223-234.

29. Sorensen AG, Buonanno FS, Gonzalez RG, et al. Hyperacute stroke: evaluation with combined multisection diffusion-weighted and hemodynamically weighted echo-planar MR imaging. Radiology 1996; 199: 391-401.

30. Lutsep HL, Albers GW, DeCrespigny A, Kamat GN, Marks MP, Moseley ME. Clinical utility of diffusion-weighted magnetic resonance imaging in the assessment of ischemic stroke. Ann Neurol 1997; 41: $574-580$.

31. Zelaya F, Flood N, Chalk JB, et al. An evaluation of the time dependence of the anisotropy of the water diffusion tensor in acute human ischemia. Magn Reson Imaging 1999; 17: 331-348.

32. Weber J, Mattle HP, Heid O, Remonda L, Schroth G. Diffusionweighted imaging in ischemic stroke: a follow-up study. Neuroradiology 2000; 42: 184-191.

33. Yang Q Tress BM, Barber PA, et al. Serial study of apparent diffusion coefficient and anisotropy in patients with acute stroke. Stroke 1999; 30: 2382-2390.

34. Mukherjee P, Bahn MM, McKinstry RC, et al. Differences between gray matter and white matter diffusion in stroke: diffusion-tensor MR imaging in 12 patients. Radiology 2000; 215: 211-220.

35. Copen WA, Schwamm LH, Gonzalez RG, et al. Ischemic stroke: effects of etiology and patient age in the time course of the core apparent diffusion coefficient. Radiology 2001; 221: 27-33.

36. Sorensen AG, Wu O, Copen WA, et al. Human acute cerebral ischemia: detection of changes in water diffusion anisotropy by using MR imaging. Radiology 1999; 212: 785-792.

37. Werring DJ, Toosy AT, Clark CA, et al. Diffusion tensor imaging can detect and quantify corticospinal tract degeneration after stroke. J Neurol Neurosurg Psychiatry 2000; 69: 269-272.

38. Schlaug G, Siewert B, Benfield A, Edelman RR, Warach S. Time course of the apparent diffusion coefficient (ADC) abnormality in human stroke. Neurology 1997; 49: 113-119.

39. Gideon P, Stahlberg F, Thomsen C, Gjerris F, Sorensen PS, Henriksen $\mathrm{O}$. Cerebrospinal fluid flow and production in patients with normal pressure hydrocephalus studied by MRI. Neuroradiology 1994; 36: 210-215.

40. Hachinski VC, Potter P, Merskey H. Leukoaraiosis. Arch Neurol 1987; 44: 21-33.

41. Brown MM. Leukoaraiosis. In: Donnan GA, Norrving B, Bamford JM, Bogousslavsky J (eds) Lacunar and Other Subcortical Infarctions. Oxford University Press, Oxford, pp. 181-198, 1995.

42. Pantoni L, Garcia JH. Pathogenesis of leukoaraiosis: a review. Stroke 1997; 28: 652-659.

43. O'Sullivan M, Summers PE, Jones DK, Jarosz JM, Willimas SC, Markus HS. Normal-appearing white matter in ischemic leukoaraiosis: a diffusion tensor MRI study. Neurology 2001; 57: 23072310.

44. Jones DK, Lythgoe D, Horsfield MA, Simmons A, Williams SCR, Markus HS. Characterization of white matter damage in ischemic leukoaraiosis with diffusion tensor MRI. Stroke 1999; 30: 393-397.

45. Pierpaoli C, Barnett A, Pajevic S, et al. Water diffusion changes in Wallerian degeneration and their dependence on white matter architecture. Neuroimage 2001; 13: 1174-1185.

46. Nell J, Shiran S, McKinstry R, et al. Normal brain in human newborns: apparent diffusion coefficient and diffusion anisotropy measured by using diffusion tensor MR imaging. Radiology 1998; 209: 57-66.

47. Wimberger DM, Roberts TR, Barkovitch AJ, Prayer LM, Moseley ME, Kucharczyk J. Identification of "premyelination" by diffusionweighted MRI. J Comput Assist Tomogr 1995; 19: 28-33.

48. Sakuma H, Nomura Y, Takeda K, et al. Adult and neonatal human brain: diffusional anisotropy and myelination with diffusion-weighted MR imaging. Radiology 1991; 180: 229-233.

49. Nomura Y, Sakuma H, Takeda K, Tagami T, Okuda Y, Nakagawa T. Diffusion anisotropy of the human brain assessed with diffusionweighted MR: relation with normal brain development and aging. AJNR Am J Neuroradiol 1994; 15: 231-238.

50. Huppi P, Maier S, Peled S, et al. Microstructural development of human newborns cerebral white matter assessed in vivo by diffusion tensor MRI. Pediatr Res 1998; 44: 584-590.

51. Klingberg T, Vaidya CJ, Gabrieli JDE, Moseley ME, Hedehus M. Myelination and organization of the frontal white matter in children: a diffusion tensor MRI study. Neuroreport 1999; 10: 2817-2821.

52. Nusbaum AO, Lu D, Tang CY, Altas SW. Quantitative diffusion measurements in focal multiple sclerosis lesions: correlations with appearance on T1-weighted images. AJNR Am J Neuroradiol 2000; 175: 821-825.

53. Gideon $\mathrm{P}$, Thomsen $\mathrm{C}$, Henriksen O. Increased self-diffusion of brain water in normal aging. Magn Reson Imaging 1994; 4: 185-188.

54. O'Sullivan MO, Jones DK, Summers PE, Morris RG, Williams SC, Markus HS. Evidence for cortical "disconnection" as a mechanism of age-related cognitive decline. Neurology 2001; 57: 632-638.

55. Pfefferbaum A, Sullivan EV, Hedehus M, Adalsteinsson E, Lim KO, Moseley M. In vivo detection and functional correlates of white matter microstructural disruption in chronic alcoholism. Alcohol Clin Exp Res 2000; 24: 1214-1221.

56. Pfefferbaum A, Rosenbloom MJ, Serventi K, Sullivan EV. Corpus callosum, pons and cortical white matter in alcoholic women. Alcohol Clin Exp Res 2002; 26: 400-405.

57. Arfanakis K, Haughton VM, Carew JD, Rogers BP, Dempsey RJ, Meyerand ME. Diffusion tensor MR imaging in diffuse axonal injury. AJNR Am J Neuroradiol 2002; 23: 794-802.

58. Huisman TAGM, Schwamm LE, Schaefer PW, et al. Diffusion tensor imaging as potential biomarker of white matter injury in diffuse axonal injury. AJNR Am J Neuroradiol 2004; 25: 370-376.

59. Strich SJ. Shearing of nerve fibres as a cause of brain damage due to head injury: a pathological study of twenty cases. Lancet 1961; 2: 443-448.

60. Adams JH, Graham DI, Murray LS, Scott G. Diffuse axonal injury due to nonmissile head injury in humans: an analysis of 45 cases. Ann Neurol 1982; 12: 557-563. 
61. Huisman AGM, Sorensen AG, Hergan K, Gonzalez RG, Schaefer PW. Diffusion-weighted imaging for the evaluation of diffuse axonal injury in closed head injury. J Comp Assist Tomogr 2003; 27: 511.

62. Liu AY, Maldjian JA, Bagley LJ, Sinson GP, Grossman RI. Traumatic brain injury. AJNR Am J Neuroradiol 1999; 20: 16361641.

63. Jones DK, Daris R, Erivne M, et al. Strong AJ. Cluster analysis of diffusion tensor magnetic resonance images in human head injury. Neurosurgery 2000; 47: 306-317.

64. Mckinstry RC, Miller JH, Snyder AZ, et al. A prospective, longitudinal diffusion tensor imaging study of brain injury in newborns. Neurology 2002; 59: 824-833.

65. Brunberg J, Chenevert T, McKeever PE, et al. In vivo MR determination of water diffusion coefficients and diffusion anisotropy: correlation with structural alteration in gliomas of the cerebral hemispheres. AJNR Am J Neuroradiol 1995; 16: 361-371.

66. Krabbe K, Gideon P, Wagn P, Hansen U, Thomsen C, Madsen F. MR diffusion imaging of human intracranial tumors. Neuroradiology 1997; 39: 483-489.

67. Castillo M, Smith J, Kwock L, Wilbert K. Apparent diffusion coefficients in the evaluation of high-grade cerebral gliomas. ANJR Am J Neuroradiol 2001; 22: 60-64.

68. Guo A, Cummings T, Dash R, Provenzale J. Lymphomas and highgrade astrocytomas: comparison of water diffusibility and histologic characteristics. Radiology 2002; 224: 177-183.

69. Sinha S, Bastin ME, Whittle IR, Wardlaw JM. Diffusion tensor imaging of high-grade cerebral gliomas. AJNR Am J Neuroradiol 2002; 23: 520-527.

70. Bastin M, Sinha S, Whittle I, Wardlaw J. Measurements of water diffusion and $\mathrm{T} 1$ values in peritumoral oedematosus brain. Neuroreport 2002; 13: 1335-1340

71. Lu S, Ahn D, Johnson G, Law M, Zagzag D, Grossman RI. Diffusion-tensor MR imaging of intracranial neoplasia and associated peritumoral edema: introduction of the tumor infiltration index. Radiology 2004; 232: 221-228.

72. Tropine A, Vucurevuc G, Delani P, et al. Contribution of diffusion tensor imaging to delineation of gliomas and glioblastomas. J Magn Reson Imaging 2004; 20: 905-912.

73. Prince SJ, Burnet NG, Donovan T, et al. Diffusion tensor imaging of brain tumors at 3T: a potential tool for assessing white matter tract invasion? Clin Radiol 2003; 58: 455-462.

74. Lu S, Ahn D, Johnson G, Cha S. Peritumoral diffusion tensor imaging of high-grade gliomas and metastatic brain tumors. AJNR Am J Neuroradiol 2003; 24: 937-941.

75. Witmer BP, Moftakhar R, Hasan KM, et al. Diffusion-tensor imaging of white matter tracts in patients with cerebral neoplasm. J Neurosurg 2002; 97: 568-575.

76. Wieshmann UC, Symms MR, Parker GJ, et al. Diffusion tensor imaging demonstrates deviation of fibers in normal appearing white matter adjacent to brain tumor. J Neurol Neurosurg Psychiatry 2000; 68: 501-503.

77. Gauvain K, McKinstry R, Mukherjee P, et al. Evaluating pediatric brain tumor cellularity with diffusion tensor imaging. AJR Am J Roentgenol 2001; 177: 449-454.

78. Mori S, Fredriksen K, van Zijl PC, et al. Brain white matter anatomy of tumor patients evaluated with diffusion tensor imaging. Am Neurol 2002; 51: 377-380.

79. Field AS, Alexander AL, Hasan KM, et al. Diffusion tensor MR imaging patterns in white matter tracts altered by neoplasm. Presented at the 10th meeting of the International Society of Magnetic Resonance in Medicine Workshop on Diffusion MRI Biophysical Issues, St Malo, France, 10-12 March, 2002.

80. Chenevert TL, McKeever PE, Ross BD. Monitoring early response of experimental brain tumors to therapy using diffusion magnetic resonance imaging. Clin Cancer Res 1997; 3: 1457-1466.
81. Chenevert TL, Stegman LD, Taylor JM et al.. Diffusion magnetic resonance imaging; an early surrogate marker of therapeutic efficacy in brain tumors. J Natl Cancer Inst 2000; 92: 2029-2036.

82. Mardor Y, Pfeffer R, Spiegelmann R, et al. Early detection of response to radiation therapy in patients with brain malignancies using conventional and high b-value diffusion weighted magnetic resonance imaging. J Clin Oncol 2003; 21: 1094-1100.

83. Jäger HR, Waldman AD, Benton C, Fox N, Rees J. Differential chemosensitivity of tumor components in malignant oligodendroglioma: assessment with diffusion-weighted, perfusion-weighted, and serial volumetric MR imaging. AJNR Am J Neuroradiol 2005; 26: 274-278.

84. Bonavita S, Di Salle F, Tedeschi G. Proton MRS in neurological disorders. Eur Radiol 1999; 30: 125-131.

85. Dong Q Sundgren PC, Weybright P, Mukherji S, Welsh RC, Chenevert TL. Differentiation of Tumor Recurrence Form Radiation-induced Necrosis using Diffusion Tensor Imaging. ISMRM 2004 Scientific Exhibit. SE2076 Proceedings, p. 394, 2004.

86. Hein PA, Eskey CJ, Dunn JF, Hug EB. Diffusion-weighted imaging in the follow-up of treated high-grade gliomas: tumor recurrence versus radiation injury. AJNR Am J Neuroradiol 2004; 25: 201-209.

87. Le Bihan D, Douek P, Argyropoulou M, Turner R, Patronas N, Fulham M. Diffusion and perfusion magnetic resonance imaging in brain tumors. Top Magn Reson Imaging 1993; 5: 25-31.

88. Tung GA, Evangelista P, Rogg JM, Duncan JA III. Diffusionweighted MR imaging of rim-enhancing brain masses: is markedly decreased water diffusion specific for brain abscess. AJR Am J Roentgenol 2001; 177: 709-712.

89. Biousse V, Newman NJ, Hunter SB, Hudgins PA. Diffusion weighted imaging in radiation necrosis. J Neurol Neurosurg Psychiatry 2003; 74: 382-784.

90. Zhou XJ, Leeds NE, Kumar AJ, Chong J, Levin VA. Differentiation of tumor recurrence from treatment-induced necrosis using quantitative diffusion MRI. Proc Int Soc Magn Reson Med 2001; 9: 726.

91. Sundgren PC, Fan XY, Dong Q, Webright P, Welsh RC, Chenevert TL. Discriminating brain tumor recurrence from radiation-induced injury using diffusion tensor imaging. Unpublished Data to be Presented at the ASNR 43rd Annual Meeting, Toronto, May 23-27, 2005.

92. Droogan AG, Clark CA, Werring DJ, Barker GJ, McDonald WI, Miller DH. Comparison of MS clinical subgroups using navigated diffusion-weighted imaging. Magn Reson Imaging 1999; 17: 653661.

93. Werring DJ, Clark CA, Barker GJ, Thompson AJ, Miller DH. Diffusion tensor imaging of lesions and normal-appearing white matter in multiple sclerosis. Neurology 1999; 52: 1626-1632.

94. Bammer R, Augustin M, Strasser-Fuchs S, et al. Magnetic resonance diffusion tensor imaging for characterizing diffuse and focal white matter abnormalities in multiple sclerosis. Magn Reson Med 2000; 44: 583-591.

95. Filippi M, Ianucci G, Cercignani M, Rocca MA, Praseti A, Comi G. A quantitative study of water diffusion in multiple sclerosis lesions and normal-appearing white matter using echo-planar imaging. Arch Neurol 2000; 57: 1017-1021.

96. Bozzali M, Cercignani M, Sormani M, Comi G, Filippi M. Quantification of brain gray matter damage in different MS phenotypes by use of diffusion tensor MR imaging. AJNR Am J Neuroradiol 2002; 23: 985-988.

97. Rovaris M, Iannucci G, Falautano M, et al. Cognitive dysfunction in patients with mildly disabling relapsing-remitting multiple sclerosis; an exploratory study with diffusion tensor MR imaging. J Neurol Sci 2002; 195: 103-109.

98. Guo A, MacFall J, Provenzale J. Multiple sclerosis; diffusion tensor MR imaging for evaluation of normal-appearing white matter. Radiology 2002; 222: 729-736. 
99. Filippi M, Cercignani M, Inglese M, Horsfield M, Comi G. Diffusion tensor magnetic resonance imaging in multiple sclerosis. Neurology 2001; 56: 304-311.

100. Brown WJ, Babb TL. Neuropathological changes in the temporal lobe associated with complex partial seizures. In: Hopkins A (ed.) Epilepsy. Demos, New York, NY, 1987; 300-323.

101. Brooks B, King D, Gammal T, et al. MR imaging in patients with intractable complex partial epileptic seizures. AJNR Am J Neuroradiol 1990; 11: 93-99.

102. Tien R, Felsberg G, Castro C, et al. Complex partial seizure and mesial temporal sclerosis: evaluation with fast spin-echo MR imaging. Radiology 1993; 189: 835-842.

103. Wieshman UC, Clark CA, Symms MR, Franconi F, Barker GJ, Shorvon SD. Reduced anisotropy of water diffusion in human hippocampus in epilepsy. Magn Reson Imaging 1999; 17: 29-36.

104. Yoo SY, Chang KH, Song IC, et al. Apparent diffusion coefficient value of the hippocampus in patients with hippocampal sclerosis and in healthy volunteers. AJNR Am J Neuroradiol 2002; 23: 809-812.

105. Gomez-Hassan DM, Dong Q, Maly-Sundgren PC, Milling C. Diffusion tensor imaging of cortical lesions in patients with refractory epilepsy. In: Proceedings ASNR, 2003, 188 pp. (SS 236).

106. Eriksson SH, Rugg-Gunn FJ, Symms MR, Barker GJ, Duncan JS. Diffusion tensor imaging in patients with epilepsy and malformations of cortical development. Brain 2001; 124; 617-626.

107. Arfanakis K, Hermann BP, Rogers BP, Carew JD, Seidenberg M, Meyerand ME. Diffusion tensor MRI in temporal lobe epilepsy. Magn Reson Imaging 2002; 20: 511-519.

108. Rugg-Gunn F, Eriksson S, Symms M, et al. Diffusion tensor imaging of cryptogenic and acquired partial epilepsies. Brain 2001; 124: 627636.

109. Lim CCT, Yin H, Kun Loh N, Chua VGE, Hui F, Barkowich AJ. Malformations of cortical development: high-resolution MR and diffusion tensor imaging of fiber tracts at 3T. AJNR Am J Neuroradiol 2005; 26: 61-64.

110. Albayram S, Melhem ER, Mori S, et al. Holoprosencephaly in children; diffusion tensor MR imaging of white matter tracts of the brainstem: initial experience. Radiology 2002; 223: 645-651.

111. Hanyu H, Sakurai H, Iwamoto T, Takasaki M, Shindo H, Abe K. Diffusion-weighted MR imaging of the hippocampus and temporal white matter in Alzheimer's disease. J Neurol Sci 1998; 156: 195200.

112. Rose SE, Chen F, Chalk JB, et al. Loss of connectivity in Alzheimer's disease: an evaluation of the white matter tract integrity with color coded MR diffusion tensor imaging. J Neurol Neurosurg Psychiatry 2000; 69: 528-530.

113. Klingberg T, Hedehus M, Temple E, et al. Microstructure of temporo-parietal white matter as a basis for reading ability: evidence from diffusion tensor magnetic resonance imaging. Neuron 2000; 25 : 493-500.

114. Molko N, Cohen L, Mangin JF, et al. Visualizing the neural bases of a disconnection syndrome with diffusion tensor imaging. J Cogn Neurosci 2002; 14: 629-636.

115. Peng SS-F, Tseng W-YI, Chien Y-H, Hwu W-L, Liu H-M. Diffusion tensor imaging in children with early-treated, chronic, malignant phenylketonuric: correlation with intelligence assessment. AJNR Am J Neuroradiol 2004; 25: 1569-1574.

116. Itoh R, Melhem ER, Mori S, Eichler FS, Raymond GV, Moser HW. Diffusion tensor brain MR imaging in X-linked cerebral adrenoleukodystrophy. Neurology 2001; 56: 544-547.

117. Eichler F, Itoh R, Barker PB, et al. Proton MR spectroscopic and diffusion tensor brain MR imaging in X-linked adrenoleukodystrophy: initial experience. Radiology 2002; 225: 245-252.

118. Schneider JFL, Il'yasov KA, Boltshauser E, Hennig J, Martin E. Diffusion tensor imaging in cases of adrenoleukodystrophy: preliminary experience as a marker for early demyelination? AJNR Am J Neuroradiol 2003; 24: 819-824.

119. Guo AC, Petrella JR, Kurtzberg J, Provenzale JM. Evaluation of white matter anisotropy in Krabbe disease with diffusion tensor imaging: initial experience. Radiology 2001; 218: 245-252.

120. Ono J, Harada K, Takahashi M, Maeda M, et al. Differentiation between dysmyelination and demyelination using magnetic resonance diffusional anisotropy. Brain Res 1995; 671: 141-148.

121. Ono J, Harada K, Mano T, Sakurai K, Okada S. Differentiation of dys- and demyelination using diffusional anisotropy. Pediatr Neurol 1997; 16: 63-66.

122. Schaumburg H, Powers J, Raine C, Suzuki K, Richardson E. Adrenoleukodystrophy: a clinical and pathological study of 17 cases. Arch Neurol 1975; 32: 577-591. 